\title{
MODELACIÓN ECONÓMICA CON INFORMACIÓN ESPACIALMENTE EXPLÍCITA DE LA DEFORESTACIÓN EN URABÁ, COLOMBIA, 1980-2000*
}

\author{
Cristian David Ramírez Sosa** \\ Sergio Alonso Orrego Suaza***
}

Recibido: septiembre 09 de 2010

Aceptado: septiembre 02 de 2011

\section{RESUMEN}

La deforestación tropical contribuye al cambio climático por ser la segunda fuente neta de emisión a la atmósfera de gases con efecto de invernadero. El objetivo del artículo fue la identificación de la importancia relativa de los determinantes de la deforestación observada en Urabá, 1980-2000. Para ello se usaron modelos conceptuales basados en teoría económica del uso de la tierra rural, junto con modelos estadísticos discretos dicotómicos en los que se empleó información espacialmente explícita. Los resultados indican que la especialización en pasto, la especialización en maíz, la distancia a centros de importancia regional y la distancia a los principales ríos de la región fueron los principales determinantes de la deforestación en Urabá. Información sobre la importancia relativa de los determinantes de la deforestación, constituye información valiosa para el diseño de política pública y proyectos de cambio climático en Colombia.

\section{PALABRAS CLAVE}

Deforestación tropical, cambio climático, modelación económica, sistemas de información geográfica, Urabá-Colombia.

\section{CLASIFICACIÓN JEL}

C51; Q15; R14

\section{CONTENIDO}

Introducción; 1. Revisión de literatura; 2. Métodos; 3. Resultados; 4. Discusión; 5. Conclusiones; Bibliografía.

Artículo de investigación producto del trabajo de grado de Cristian David Ramírez Sosa, para optar al título de Ingeniero Forestal en la Universidad Nacional de Colombia, sede Medellín. El trabajo de grado fue un compromiso académico en el proyecto de investigación Costos de captura de carbono a través de actividades de forestación en Colombia (código 070302020), financiado por la Dirección de Investigación de la Universidad Nacional de Colombia, Sede Medellín, y ejecutado entre agosto de 2007 y febrero de 2010. El proyecto de investigación se formuló y ejecutó por el grupo de investigación Bosques y Cambio Climático, clasificado en la categoría B por Colciencias.

* Ingeniero Forestal, Universidad Nacional de Colombia, Sede Medellín. Estudiante de la Maestría en Medio Ambiente y Desarrollo de la Facultad de Minas, Universidad Nacional de Colombia, sede Medellín. Calle 59 BB No 68 C 40, Apto. 201, Bello, Colombia. Teléfono (57 4) 4560813. Correo electrónico: cdramire@unal.edu.co.

*.* Ingeniero Forestal, Universidad Nacional de Colombia, Sede Medellín. MSc Economía de los Recursos Naturales y del Medio Ambiente, Universidad de Concepción, Chile. PhD en Recursos Forestales, Oregon State University, Estados Unidos. Profesor Departamento de Ciencias Forestales, Universidad Nacional de Colombia, sede Medellín, Colombia. Miembro del grupo de investigación en Bosques y Cambio Climático, Universidad Nacional de Colombia, Sede Medellín. Calle 59 A No 63-020 Sector Otra banda, Bloque 14, Apartado Aéreo 1027, Medellín, Colombia. Teléfono (57-4) 4309085. Correo electrónico: saorrego@unal.edu.co. 


\section{ECONOMIC MODELING WITH SPECIALLY EXPLICIT INFORMATION ON DEFORESTATION IN URABA, COLOMBIA 1980-2000}

\section{ABSTRACT}

Tropical deforestation contributes to climate change due to the fact that it's the second source of greenhouse gas net emissions. The objective of this article is to identify the relative importance of the deforestation determinants that were observed in Urabá, 1980-2000. Conceptual models based on economic theory on rural land use where used together with discrete dichotomy statistic models in were the especially explicit information was used. The results indicate that specialization on pastures, corn, the distance from the main regional centers and main rivers of the region where the main determinants of deforestation in Urabá. The information about the relative importance of the deforestation determinants constitutes valuable information for designing public policies and climate change projects in Colombia.

\section{KEY WORDS}

Tropical deforestation, climate change, economic modeling, geographic information systems, Urabá-Colombia.

\section{JEL CLASSIFICATION}

C51; Q15; R14

\section{CONTENT}

Introduction; .1 Literature review; 2. Methods; 3. Results; 4. Discussion; 5. Conclusions; Bibliography.

\section{MODELAÇÃO ECONÔMICA USANDO INFORMAÇÃO ESPECIALMENTE EXPLICITA DA DEFLORESTAÇÃO EM URABÁ, COLÔMBIA 1980-2000}

\section{RESUMO}

A deflorestação tropical contribui as alterações climáticas dado que é a segunda fonte neta de emissão à atmosfera de gases com efeito de estufa. O objetivo do artigo foi identificar a importância relativa dos determinantes de deflorestação observada em Urabá, 19802000. Para este objetivo, usaram-se modelos conceptuais baseados na teoria econômica da utilização de terra rural, junto com modelos estatísticos discretos dicotômicos nos que empregou-se a informação especialmente explicita. Os resultados indicam que a especialização nos pastagens, a especialização em milho, a distancia aos centros de importância regional e a distancia aos principais rios da região, foram as principais determinantes da deflorestação em Urabá. Informação sobre a importância relativa dos determinantes da deflorestação constitui informação valiosa para desenhar políticas públicas e projetos em alterações climáticas na Colômbia.

\section{PALAVRAS CHAVE}

Deflorestação tropical, alterações climáticas, modelação econômica, sistemas de informação geográfica, Urabá-Colômbia.

\section{CLASSIFICAÇÃO JEL} C51; Q15; R14

\section{CONTEÚDO}

Introdução; 1. Revisão de literatura; 2. Métodos; 3. Resultados; 4. Discussão; 5. Conclusões; Bibliografia. 


\section{INTRODUCCIÓN}

La deforestación tropical contribuye al cambio climático global y representa, después de la quema de combustibles fósiles, la segunda fuente neta de emisión a la atmósfera de gases con efecto invernadero (GEI) (Van der Werf y otros, 2009). Esta consecuencia ambiental de la deforestación tropical podría explicar la cantidad creciente de estudios sobre los principales determinantes biofísicos y socio-económicos de la conversión de bosques tropicales (Pfaff, 1999; Geist y Lambin, 2002; Chomitz y Thomas, 2003; Aguiar, Câmara y Escada, 2007). No obstante, los resultados de estudios previos sugieren que la importancia relativa de los determinantes de la deforestación varía según el contexto geográfico, económico e institucional. Además, la mayoría de los estudios de deforestación tienden a usar información espacialmente explícita a una escala gruesa ( $>$ 1:60.000), lo cual podría ser inapropiado para identificar los mecanismos que explican la deforestación en sitios específicos y a una escala semifina ( 1:25.000).

De acuerdo con un estudio reciente de deforestación en Antioquia, realizado por Orrego (2009), en el cual se empleó información de coberturas terrestres a escala 1:25.000 y variables explicadoras de la deforestación para un área relativamente extensa ( 6,2 millones de ha), 84.278 ha de bosques que existían en 1980 en la región de Urabá se convirtieron a usos alternativos, veinte años después. Ello probablemente como resultado de la ocurrencia simultánea de procesos incontrolados de colonización y de la expansión y consolidación de agricultura comercial, representada fundamentalmente por la producción de banano. Sin embargo, la compleja coexistencia de determinantes de deforestación en el ámbito local, podría no capturarse con un modelo de deforestación estimado para todo el departamento de Antioquia.

Un modelo conceptual basado en un mercado por tierras sin cobertura forestal se empleó en la identificación de la importancia relativa de los determinantes de la deforestación observada en Urabá, Colombia, en el período 1980-2000. El área de estudio es la zona centro y norte de Urabá, la cual incluye los siguientes municipios: Apartadó, Arboletes, Carepa, Chigorodó, Mutatá, Necoclí, San Juan de Urabá, San Pedro de Urabá y Turbo. Los determinantes se asocian con variables de aptitud agrícola, accesibilidad a mercados o condiciones socioeconómicas. Los potenciales determinantes de la deforestación se incorporaron como información espacialmente explícita a modelos estadísticos de naturaleza dicotómica, para estimar la probabilidad de deforestación de un píxel cubierto con bosque, la unidad de análisis en la presente investigación.

La magnitud de los parámetros estimados y los valores de elasticidades sugieren que los principales determinantes de la deforestación en Urabá fueron la especialización en pasto, la especialización en maíz, la distancia ponderada por la impedancia ${ }^{1}$ a los centros de importancia regional y la distancia a los principales ríos de la región. Los parámetros con menor valor en magnitud correspondieron a la densidad poblacional en 1990, así como a la distancia a centros de importancia regional y a los principales ríos. El modelo estadístico seleccionado se empleó para obtener una superficie continua de probabilidades estimadas de deforestación para toda el área cubierta por bosques en 1980. Investigaciones sobre los determinantes locales de la deforestación tropical podrían proporcionar información valiosa para la formulación de políticas públicas orientadas a atenuar la deforestación y el cambio climático.

Como hipótesis de la presente investigación se plantea que los bosques en la región de Urabá se deforestaron como consecuencia de la coexistencia de múltiples factores asociados a la expansión de la agricultura comercial, la producción agrícola de

Esta distancia equivale a la identificación sobre una superficie de la ruta de menor costo desde un píxel a distintos mercados definidos por jerarquías de centros poblados. Detalles del cálculo de la variable se presenta en la sección de Métodos. 
subsistencia y la existencia de vías que garantizaron accesibilidad a las tierras forestales. Ello contrasta con patrones secuenciales de deforestación tropical, en los que la conversión de bosques se explica inicialmente por cultivos de subsistencia y luego por la expansión de agricultura comercial. El objetivo principal del trabajo es modelar la deforestación en Urabá desde una perspectiva económica con información espacialmente explícita, e identificar la importancia relativa de los principales determinantes de la pérdida de bosques naturales en un período de veinte años.

El artículo se organiza de la siguiente manera. En la primera sección se presenta la revisión de literatura, en la cual se resumen estudios previos e importantes de deforestación tropical, realizados en Suramérica y Colombia. En la segunda sección se presenta el marco conceptual de la deforestación, basado en teorías económicas del uso de la tierra rural, el área de estudio y los métodos usados para la estimación de modelos econométricos de deforestación. En la tercera y cuarta secciones se presentan los resultados y la discusión, respectivamente. Finalmente, se presentan las principales conclusiones.

\section{REVISIÓN DE LITERATURA}

La deforestación tropical constituye una preocupación científica debido a su magnitud y efecto en el calentamiento global del planeta. La deforestación tropical, mediante la arraigada práctica de aprovechar unas pocas especies forestales con valor comercial y la posterior quema de la cobertura vegetal remanente, es la segunda fuente neta de emisión a la atmósfera de gases con efecto de invernadero (GEI), 1,2 PgC en el período 1997-2006 (Van der Werf y otros, 2009). La emisión de GEI por deforestación es superada únicamente por las emisiones de gases derivados de la quema de combustibles fósiles. Los científicos intentan explicar y modelar la deforestación mediante aproximaciones metodológicas propias de disciplinas como la geografía, la ecología del paisaje o la economía.

Desde una perspectiva económica, Kaimowitz y Angelsen (1998) hacen un recuento de varios modelos que se han propuesto para el análisis de la deforestación tropical, en los cuales se incluyen modelos estadísticos en los que el cambio de coberturas terrestres se estudia con información georreferenciada o espacialmente explícita. La evidencia empírica indica que la deforestación tropical es un proceso complejo y dinámico, influenciado por variables demográficas (densidad y distribución de la población), económicas (mercados de productos agrícolas, ingreso per-cápita, acceso al crédito en áreas rurales), políticas públicas, cambios tecnológicos y valores culturales (Geist y Lambin, 2002).

En los estudios de deforestación tropical se emplean diferentes fuentes de información de coberturas terrestres: inventarios forestales, imágenes de satélite y fotografías aéreas. Sin embargo, en áreas tropicales es muy difícil asegurar la disponibilidad de información confiable y consistente de coberturas terrestres y de los determinantes de su cambio. Por tanto, uno de los desafíos de los estudios de deforestación tropical consiste en la identificación de variables proxies para los principales determinantes de la deforestación.

Una revisión exhaustiva de más de 150 modelos económicos de deforestación tropical, realizada por Geist y Lambin (2002), indica que bosques muy próximos a vías tienen una mayor probabilidad de ser deforestados. Ello también ocurre en bosques ubicados muy cerca a áreas urbanas, o en climas secos y sitios con topografía relativamente plana, buen drenaje natural y alta fertilidad natural. Los resultados de los modelos sugieren también que los bosques fragmentados poseen un riesgo mayor de conversión, si se comparan con bosques representados espacialmente como áreas compactas. La existencia de vías y algunas características ambientales actúan de manera sinérgica en la conversión de bosques tropicales. Esto explicaría una mayor deforestación en sitios atravesados por vías, con 
suelos fértiles y climas aptos para la agricultura (Chomitz y Gray, 1996; Kaimowitz y Angelsen, 1998).

Entre los estudios de deforestación hechos en Suramérica, es importante mencionar el de Pfaff (1999) en la Amazonia brasileña. En este estudio se combinaron datos de coberturas terrestres, provenientes de imágenes de satélite, con datos socioeconómicos para cada municipio para identificar los principales determinantes de la deforestación. En Colombia, los estudios de deforestación se han concentrado en las tierras bajas de la Amazonia (Armenteras y otros, 2006; Etter y otros, 2006a; Etter y otros, 2006b; Etter y otros, 2006c; Etter y otros, 2006d), con métodos basados esencialmente en la ecología del paisaje. En el departamento de Antioquia se realizó recientemente un estudio a una escala semi-fina (1:25.000), en el que tanto la teoría de la renta como un modelo económico de uso de la tierra rural se emplearon para examinar los determinantes de la deforestación observada en el período 1980-2000 (Orrego, 2009). En este estudio se encontró que un tamaño de píxel de $30 \mathrm{~m}$ es el más adecuado para la representación en formato raster de la variable de deforestación y de aquellas que explican su ocurrencia. Los resultados indican que los principales determinantes de la deforestación en Antioquia fueron el índice de Gini, que mide la concentración de la propiedad de la tierra rural, la pendiente y la distancia a vías principales. Los resultados del estudio en Antioquia sugieren que en el noroeste del departamento, los bosques se deforestaron probablemente como consecuencia de la expansión de la agricultura comercial, principalmente plantaciones de banano, y la existencia de vías con muy buenas condiciones de transitabilidad (Orrego, 2009).

\section{MÉTODOS}

\subsection{Marco conceptual de la deforestación}

El modelo económico conceptual que se empleó en la investigación se basa en teorías económicas del uso de la tierra rural. Se asume que una parcela de tierra se dedicará al uso que proporcione el mayor valor descontado del flujo de beneficios netos (Chomitz y Gray, 1996; Lubowski y otros, 2006). El beneficio neto o renta que se deriva de un uso específico de la tierra rural está determinado fundamentalmente por características que afectan la productividad agrícola (pendiente, fertilidad del suelo y clima) y el acceso a mercados (distancias a centros urbanos). En lo que respecta al acceso, la existencia de vías y la calidad de estas influencian significativamente los costos de transporte de productos agrícolas y, por consiguiente, los retornos económicos del uso de la tierra rural (Chomitz y Gray, 1996; Nelson y Hellerstein, 1997; Pfaff, 1999).

El propietario o administrador de la tierra seleccionará un uso al comparar el valor presente neto de la renta de todos los usos alternativos. A continuación se describe el modelo teórico propuesto por Nelson y Hellerstein (1997), que permite entender las decisiones de uso de la tierra rural. La renta derivada de una parcela de tierra rural $j$ que se dedica al uso $k$ en el tiempo $\mathrm{T}$, denotada por $\mathrm{R}_{\mathrm{ikT}}$ y equivalente al valor presente neto del flujo de beneficios, se representa como

$$
R_{j k T}=\int_{t=0}^{\infty}\left(P_{j k T+t} Q_{j k T+t}-C_{j k T+t} X_{j k T+t}\right) e^{-i k t} d t,
$$

donde $\mathrm{P}_{\mathrm{j} k \mathrm{~T}}$ es un vector de precios de productos, $\mathrm{O}_{i k T}$ es la cantidad de producto, $\mathrm{C}_{i k T}$ es un vector de precios de insumos, $\mathrm{X}_{\mathrm{jkT}}$ son las cantidades óptimas de insumos y $e^{-i k t}$ es el factor de descuento, en el que $i$ es la tasa de descuento específica para un sitio. El propietario seleccionará para cada parcela aquel uso de la tierra que proporcione el mayor valor de $\mathrm{R}_{j k \mathrm{~T}}$. El modelo es consistente con el que proponen Cropper, Griffiths y Mani (1999) para estudiar decisiones de conversión de tierras forestales, en el que se asume un equilibrio entre la demanda y la oferta de tierra sin cobertura forestal. Mientras la demanda por tierra sin cobertura forestal es una demanda derivada de un insumo requerido en la 
agricultura, la oferta equivale a la función inversa del costo marginal de conversión.

Según Cropper, Griffiths y Mani (1999) la demanda por tierra sin cobertura forestal o deforestada $\left(\mathrm{T}_{d}\right)$ es función del precio de la tierra deforestada $\left(p_{d}\right)$, el costo de insumos como mano de obra $(m)$ y capital $(\kappa)$, el precio de productos agrícolas $\left(p_{p a}\right)$, asî como de factores que afectan la productividad de la tierra para la agricultura, como calidad del suelo (C) y pendiente $\left(p_{e}\right)$. La versión estática del problema de maximización del beneficio neto para un agricultor se representa como

$$
\begin{aligned}
\max _{\left(m, k, T_{d}\right)} \Pi= & \left(p_{p a}-t\right) f\left(m, k, T_{d}, C, p_{e}\right) \\
& -s m-r k-p_{d} T_{d},
\end{aligned}
$$

donde $t$ representa los costos de transporte de productos agrícolas, f la función de producción, s la tasa de salario, y r el costo del capital. Las condiciones de primer orden de la solución al problema de maximización en la ecuación (2) permiten derivar la función de demanda para el insumo tierra sin cobertura forestal $\left(\mathrm{T}_{d}\right)$

$$
T_{d}=f\left(p_{p a}, t, s, r, p_{d}, C, p_{e}\right),
$$

la cual tiene como argumentos el precio de los productos agrícolas, los costos de transporte, la tasa de salario, el costo del capital, el precio de la tierra deforestada, la calidad del suelo y la pendiente. La demanda agregada por tierra sin bosques ( $T_{d}{ }^{A}$ ) se obtiene al multiplicar la ecuación (3) por el número de familias dedicadas a la agricultura (N) en un área específica, las cuales se asumen idénticas. Por tanto,

$$
T_{d}^{A}=N T_{d}\left(p_{p a}, t, s, r, p_{d}, C, p_{e}\right) .
$$

La oferta de tierras sin cobertura forestal corresponde a la función inversa del costo marginal de convertir tierras con bosques a usos alternativos. El costo marginal de conversión depende de factores fisiográficos como la pendiente, el costo de la mano de obra y otros insumos, así como de la red vial (R), la cual determina la accesibilidad a áreas con bosques. El costo marginal de conversión disminuye por el aprovechamiento de especies forestales con valor comercial, lo que constituye una fuente de ingresos determinados por el precio de las trozas de madera $\left(p_{m}\right)$. Por tanto, la función de costo marginal de conversión de tierras forestales se expresa como

$$
C=f\left(T_{d}^{s}, p_{e}, R, s, p_{m}\right),
$$

Donde $T_{d}{ }^{s}$ representa la oferta de tierra deforestada. En condiciones de equilibrio en el mercado, la cantidad de tierra deforestada $\left(\mathrm{T}_{d}\right)$ corresponde al valor en el cual la demanda y oferta se igualan ( $T_{d}{ }^{A}=T_{d}{ }^{s}$ ). La cantidad de tierra sin bosques y su respectivo precio se determinan estructuralmente por las ecuaciones (4) y (5), las cuales se estiman simultáneamente si se observara el precio de la tierra. Si no fuera así, como sucede por lo general en muchos países tropicales, es posible estimar una ecuación de la forma reducida. Por tanto, el modelo teórico sugiere que la tierra sin cobertura forestal depende del número de familias dedicadas a actividades agropecuarias $(\mathrm{N})$, los costos de transporte $(t)$, la calidad del suelo $(C)$, los precios de productos agrícolas $\left(p_{p a}\right)$, el salario $(s)$, el costo de capital $(r)$, y de variables que afectan el costo de conversión de la tierra con bosques como la red de vías (R), el precio de las trozas de madera $\left(p_{m}\right)$ y la pendiente $\left(p_{e}\right)$. Siguiendo a Cropper, Griffiths y Mani (1999), la ecuación de la cantidad de tierra forestal que se convierte a agricultura se expresa como

$$
T_{d}=f\left(N, t, C, p_{p a}, s, r, R, p_{m}, p_{e}\right) .
$$

Para el estudio de la deforestación en Urabá, se seleccionaron y calcularon variables proxies para los argumentos de la ecuación (6). La selección y el cálculo de variables fueron posibles por la existencia de datos espacialmente explícitos, obtenidos de fuentes de información como mapas básicos nacionales y bases de datos internacionales de libre acceso. Las variables empleadas en la investigación se relacionan con la accesibilidad a mercados, la aptitud agrícola de la tierra rural y condiciones socio-económicas. 
La accesibilidad a mercados se estudió con las distancias ponderadas por impedancia física $\left(d_{p i}\right)$ a cuatro tipos distintos de mercados, y la distancia euclidiana a los principales ríos $\left(d_{r}\right)$. Estas distancias determinan los costos de transporte $(t)$ y se asumieron como variables proxies para los precios de insumos $(s, r)$ y de productos agrícolas $\left(p_{p a}\right)$. La propensión natural agrícola de la tierra se analizó con la pendiente $\left(p_{e}\right)$, la zona de vida $(z)$, la aptitud agrícola $\left(a_{a}\right)$ según la clase agrológica, e índices de especialización productiva para banano $\left(e_{b}\right)$, plátano $\left(e_{p l}\right)$, maíz $\left(e_{m}\right)$ y pasto $\left(e_{p}\right)$. Como características socioeconómicas se usaron las tasas de crecimiento de la población rural por municipio para los períodos 1980-1990 $\left(t_{c 8090}\right)$ y 1990-2000 $\left(t_{c 9000}\right)$, un índice de necesidades básicas insatisfechas $\left(n_{b i}\right)$ en áreas rurales, densidad poblacional en $1990\left(d_{p}\right)$, la cual se asume como variable proxy para el número de familias dedicadas a la agricultura, así como áreas naturales protegidas $\left(a_{n p}\right)$. Por tanto,

$$
\begin{aligned}
T_{d}= & f\left(d_{p i}, d_{r}, p_{e}, z, a_{a}, e_{b}, e_{p l}, e_{m},\right. \\
& \left.e_{p}, t_{c 8090}, t_{c 9000}, n_{b i}, d_{p}, a_{n p}\right) .
\end{aligned}
$$

Varios autores (Chomitz y Gray, 1996; Cropper, Griffiths y Mani, 1999) advierten sobre la posible endogeneidad entre la población dedicada a la agricultura (N) y las vías (R). No obstante, en esta investigación se asume que las vías se construyen para la conexión de áreas remotas y que no se trazan con el propósito explícito de cruzar tierras con suelos aptos para la agricultura, situación que suele caracterizar el diseño de vías en áreas tropicales. Más que endogeneidad podría existir correlación estadística entre las variables vías y población, lo que se evaluó con el cálculo de coeficientes de correlación para todos los posibles pares de variables.

\section{2 Área de estudio}

El área de estudio es la zona centro y norte de la región de Urabá, según la definición del Departamento Administrativo de Planeación de Antioquia (figura 1). Por tanto, el área de estudio incluye nueve de los once municipios de la región de Urabá. Ellos son: Apartadó, Arboletes, Carepa, Chigorodó, Mutatá, Necoclí, San Juan de Urabá, San Pedro de Urabá y Turbo. El área de estudio tiene una superficie de $8.535 \mathrm{~km}^{2}$, equivalente a 13,8\% del área del departamento de Antioquia y una población de 500.186 habitantes de acuerdo con datos del Departamento Administrativo de Planeación y Gobernación de Antioquia (2007).

Figura 1. Localización del área de estudio: zona centro y norte de la región de Urabá.

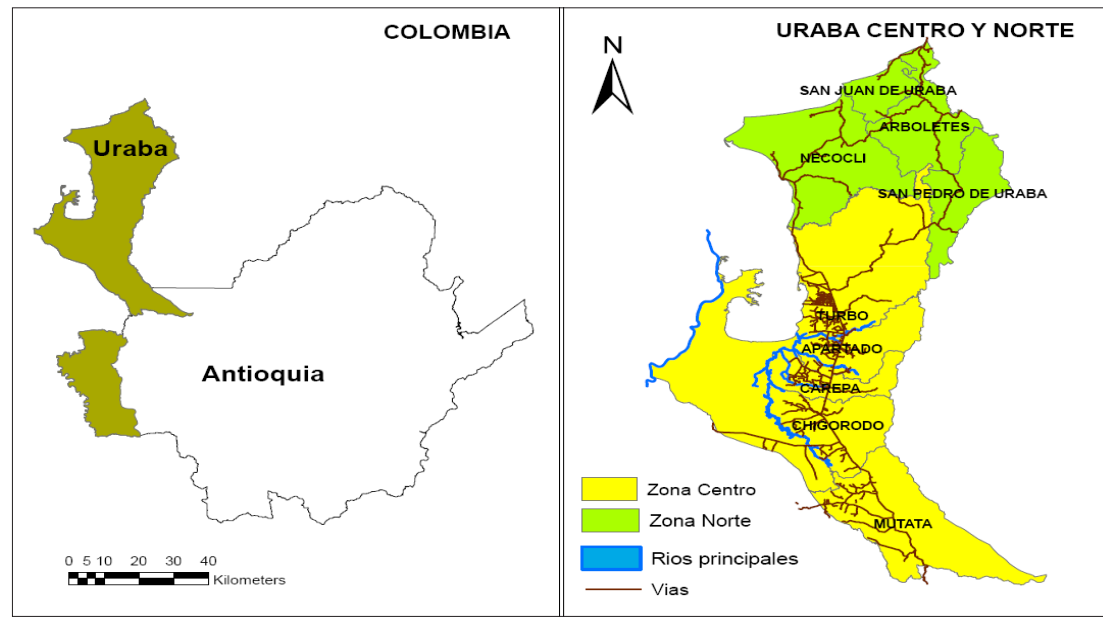

Fuente: Elaboración propia. 


\subsection{Datos y variables}

Información de coberturas terrestres de 1980 y 2000, a escala 1:25.000, se usó en la modelación de la deforestación en Urabá. El mapa de coberturas terrestres de 1980 se obtuvo de la digitalización de mapas en formato análogo proporcionados por la Secretaría de Agricultura de Antioquia (Orrego, 2009). El mapa de coberturas terrestres del año 2000 fue el resultado de un Convenio Interadministrativo entre la Secretaría de Agricultura, el Departamento Administrativo de Planeación y el Instituto Geográfico Agustín Codazzi. Ambos mapas de coberturas terrestres se convirtieron a formato raster con el uso de ArcInfo ${ }^{\mathrm{TM}}$, versión 9.0, y de ellos se identificó la trayectoria de bosque a no bosque.

Se realizó una evaluación rigurosa de la disponibilidad de información espacial, a partir de la cual se calcularon las variables explicadoras de la deforestación observada en Urabá. La información empleada en el cálculo de dichas variables se colectó de manera que representara las condiciones de acceso a mercados o aptitud agrícola que prevalecieron muy aproximadamente al inicio del período de análisis de la presente investigación. A continuación se describen brevemente las variables explicadoras.

Distancia a vías y a ríos. Las vías primarias y secundarias del área de estudio se digitalizaron en ArcGIS $^{\mathrm{TM}}$ 9.2, a partir de mapas base del año 1988, escala 1:25.000, proporcionados por la Secretaría de Agricultura. Además, se digitalizaron los dos ríos principales de la zona: el Atrato y el León. Se asume que el costo marginal de deforestación se reduce con la cercanía a vías y ríos, por lo que la correlación entre distancia a ambos atributos físicos y la deforestación se anticipa que sea negativa.

Distancias ponderadas por impedancia. La accesibilidad a mercados podría medirse objetivamente con los tiempos de viaje. Sin embargo, este tipo de información no tiende a colectarse sistemáticamente. Por ello, la mayoría de los estudios de deforestación tropical usan distancias ponderadas por la impedancia o fricción física que ejerce el tipo de vía o la pendiente (Chomitz y Gray, 1996; Nelson y Hellerstein, 1997; Mertens y Lambin, 2000; Cropper, Puri y Griffiths, 2001; Nelson y otros, 2004; Blackman y otros, 2007). Un valor de impedancia se asignó al área de estudio según las coberturas terrestres existentes en 1980. A las áreas forestales, que incluyen bosques como los cativales, manglares, mosaicos, o bosques intervenidos, y aquellas áreas cubiertas con vegetación de porte bajo, se les asignó 1 como valor de impedancia. A las áreas con uso agrícola, plantaciones forestales y urbanas, se les asignó un valor de 2. La racionalidad para ello es que las áreas sin bosques tienden a ser privadas, lo que conduce a que no sean relativamente fáciles de cruzar, comparadas con áreas boscosas que tienen derechos de propiedad no definidos o difusos. Ello es consistente con el supuesto que se asumió en el estudio de dinámica del cambio de coberturas terrestres en Honduras (Munroe, Southworth y Tucker, 2002).

A las vías pavimentadas se les asignó un valor de impedancia 1, mientras que a las no pavimentadas, un valor de 2. Ello porque las vías pavimentadas poseen mejores especificaciones técnicas, lo que reduce el tiempo de viaje a los mercados. A sitios sin vías se les asignó un valor de impedancia 4, para reflejar la dificultad en el transporte de productos agrícolas. Luego los valores de impedancia asignados se multiplicaron por una función de la pendiente, sugerida por Nelson y otros (2001). Por tanto,

$$
\left(1+\frac{p_{e}^{2}}{50}\right) * c c
$$

Donde $p$ representa la pendiente de un píxel y $c c$ el costo de cobertura terrestre, basado en el valor de impedancia. Aunque la fórmula parece un tanto arbitraria, captura una relación positiva entre una mayor pendiente y un mayor costo de accesibilidad a mercados. La función costdistance del módulo 
grid en ArcInfo ${ }^{\mathrm{TM}} 9.0$ se usó para el cálculo de la distancia acumulada de menor costo para cada píxel. Ésta equivale a la identificación sobre una superficie de la ruta de menor costo desde un píxel a distintos mercados. Estos mercados se definieron espacialmente según los tipos de centros poblados existentes en 1980: de importancia regional, secundarios, básicos y rurales (Corpourabá, 1984). Se espera que la distancia a mercados representados por centros poblados se correlacione negativamente con la deforestación.

Como centros de importancia regional se identificaron las ciudades con mayor concentración de población y de actividad económica y social en Urabá durante el período de estudio. Esas ciudades fueron Apartadó y Turbo. Como centros secundarios se consideraron Chigorodó, Currulao, Arboletes, Necoclí y San Pedro de Urabá, los cuales eran centros urbanos menores con influencia municipal y lugares de mercadeo y prestación de servicios sociales e institucionales. Como centros urbanos básicos se identificaron Carepa, Mutatá y San Juan de Urabá, los cuales eran los receptores de la actividad económica de las áreas rurales y suministraban a la población los servicios sociales e institucionales básicos. Finalmente, como centros rurales se consideraron poblados con 400 a900, habitantes, con una actividad económica predominantemente agrícola y ganadera, y una distribución minorista de la producción.

Pendiente. Se calculó un mapa raster de pendiente en grados, con base en un modelo de elevación digital de terreno con un intervalo de contorno de 30 m, obtenido del proyecto Misión Topográfica del Radar Shuttle (SRTM) (Werner, 2001), ejecutado por NASA en el año 2000. Aunque el modelo de elevación digital corresponde al último año del período de esta investigación, es apropiado para 1980 si se asume que no se observan cambios significativos en las características físicas de la tierra en un período de dos décadas. Se considera que áreas con topografía plana, cuyos suelos tienden a ser más fértiles y fácilmente mecanizables, son más aptas para la agricultura que aquellas con una topografía montañosa o escarpada. Por tanto, se anticipa una correlación negativa entre la pendiente y la deforestación.

Áreas naturales protegidas. En el área de estudio se encuentra: el Parque Nacional Natural Los Katíos, creado mediante Acuerdo 037 de 1973 con una superficie de 52000 ha, ampliado mediante Acuerdo 016 de 1979 a una superficie de 72000 ha, y declarado Patrimonio de la Humanidad en 1994, así como el Área de Reserva Biológica Natural Alto de Carepa ( 2500 ha), ubicada en el municipio de Apartadó y declarada por Corpourabá mediante Acuerdo 072 de 1996. Un archivo digital en formato vector se usó en el cálculo de una variable dicotómica o dummy para representar si un píxel corresponde o no a un área protegida. Se asume que la protección constituye un desincentivo a la deforestación, como consecuencia de potenciales demandas penales por afectación a áreas naturales legalmente protegidas.

Suelos. El mapa digital de suelos de la Secretaría de Agricultura de Antioquia del año 1979, escala 1:25.000, se empleó para la obtención de las categorías de clases agrológicas definidas por el Departamento de Agricultura de los Estados Unidos -USDA (Klingebiel y Montgomery, 1961). Con esta información se calculó una variable dummy de aptitud agrícola, con un valor de 1 para las clases agrológicas III y IV, y 0 para las restantes. Se prevé que áreas con una mayor aptitud agrícola son más susceptibles de deforestarse.

Datos demográficos. La densidad poblacional se obtuvo de un grid poblacional del mundo, con resolución espacial de 2,5 arco minutos, equivalente aproximadamente a $5 \mathrm{~km}$ en el Ecuador (CIESINCIAT, 2005). Los datos corresponden a la densidad poblacional para Colombia en 1989. Asimismo, se colectaron datos de censos nacionales de población (Gobernación de Antioquia, 2000), para el cálculo de tasas de crecimiento intercensales y decadales para la población rural y total en cada municipio 
del área de estudio. Los cambios en la población rural se asumen como una variable proxy para el desplazamiento forzado asociado al conflicto por la tierra en Urabá.

Aunque la densidad poblacional afecta tanto la demanda de productos agrícolas como el salario de la mano de obra, se asume que predomina el primer tipo de efecto. Por tanto, se espera que una mayor densidad poblacional se correlacione positivamente con la deforestación. Aumentos en la densidad y las tasas de crecimiento poblacional conducen a una mayor presión para la conversión de bosques.

Índice de especialización productiva. Se crearon archivos digitales con los índices de especialización para plátano, banano, cacao, arroz, maíz, yuca, carne y pasto, así como para el uso forestal, ganadería de doble propósito y porcicultura. Los índices fueron elaborados por Usma (1992) con base en estadísticas del sector agropecuario del año 1989. Un índice mayor a uno indica que el municipio tiende a especializarse en una actividad productiva. Se espera que la especialización en actividades extensivas como el establecimiento de pastos o el aumento en el área requerida para satisfacer una demanda creciente de producción agrícola asociada a economías campesinas o agricultura comercial conduzcan a mayor deforestación.

Indicadores socioeconómicos. Como indicadores de las condiciones de vida y el bienestar de la población se usaron el Índice de Necesidades Básicas Insatisfechas (INBI), reportado en el Anuario Estadístico del año 2000 (Gobernación de Antioquia, 2000), y el Índice de Condiciones de Vida (ICV) de 1993 (Departamento Administrativo de Planeación y Gobernación de Antioquia, 2007). El INBI identifica la proporción de personas u hogares que no tienen satisfecha alguna de las necesidades consideradas como básicas. Se asume que un INBI alto está asociado con una mayor deforestación, como resultado de una mayor presión que se ejerce sobre los bosques por una población con pocas fuentes de ingreso monetario.
Variables climáticas. Datos climáticos en formato raster y con resolución de $1 \mathrm{~km}$ en el Ecuador se obtuvieron de la base de acceso público WorldClim (Hijmans y otros, 2005). De esta base de datos se obtuvo la variable precipitación del trimestre más seco. Se asume que una mayor precipitación en el trimestre más seco se correlaciona positivamente con la deforestación, por la existencia de menores limitantes de disponibilidad de agua para la agricultura.

Zonas de vida. Una variable dummy se calculó para evaluar el efecto de la zona de vida sobre la deforestación. A un píxel ubicado en una zona de vida en el piso basal tropical se le asignó un valor de 1. Se asume que los bosques tropicales de tierras bajas son más susceptibles a deforestarse, dado que el clima y la topografía podrían ser más adecuados para la agricultura.

\subsection{Modelo econométrico de deforestación}

Los estudios de deforestación tropical con información espacialmente explícita están plagados inevitablemente de efectos de dependencia espacial, en las observaciones de la variable dependiente o en los términos del error. Algunas características biofísicas de la tierra rural tienden a exhibir autocorrelación espacial, dado que lugares muy próximos poseen tipos de suelos o valores de precipitación similares (Chomitz y Gray, 1996). La presencia de autocorrelación espacial podría llevar a que los estimadores de los parámetros en un modelo de regresión sean inconsistentes e ineficientes. Aunque existen métodos para estimar modelos de regresión espacial, estos son computacionalmente intensivos (Bell y Bockstael, 2000; Carrión-Flores y Irwin, 2004) y eficientes en tamaños de muestras relativamente pequeñas (pocos miles de observaciones), lo cual difiere del tamaño de muestra en la presente investigación (un par de millones de observaciones).

Además de la autocorrelación espacial, la multicolinealidad y la endogeneidad podrían estar 
presentes en modelos estadísticos de deforestación tropical. Para controlar la posible multicolinealidad de variables explicadoras se evaluó cómo la inclusión o exclusión de una variable específica afectaba los resultados estadísticos. La potencial endogeneidad entre deforestación y vías se controló con la inclusión de una variable que representara la aptitud agrícola, lo cual es consistente con lo realizado en la investigación de Chomitz y Gray (1996).

El posible efecto de la autocorrelación espacial se controló mediante la selección aleatoria de una muestra en la que no se incluyeron observaciones espacialmente contiguas. Para ello se utilizó el esquema de muestreo sugerido por Orrego (2009), en el que se seleccionan en ArcView ${ }^{\mathrm{TM}}$ observaciones separadas por lo menos 5 píxeles (150 m). Observaciones que correspondían a bosque en 1980 y para el 2000 se encontraban en bosque intervenido mosaico se eliminaron de la muestra. Ello porque esta trayectoria se considera degradación y no deforestación, lo cual no se modeló en la presente investigación.

La deforestación observada, definida como una variable dependiente binaria o dicotómica, se construyó al superponer los archivos digitales de coberturas terrestres de 1980 y 2000. Este archivo digital se utilizó para evaluar la posible presencia de autocorrelación espacial en la variable dependiente, mediante el cálculo del test de Morán² en ArcInfo $^{\mathrm{TM}}$ 9.0.

Los archivos en formato raster de las variables explicadoras se remuestrearon para asegurar un tamaño de píxel idéntico. Luego se calculó y analizó la matriz que contenía los coeficientes de correlación. Un coeficiente de correlación de por lo menos 0,7 se consideró como evidencia de correlación significativa entre pares de variables. Esto permitió controlar la multicolinealidad mediante la eliminación de variables muy correlacionadas (coeficiente

2 Detalles del test se encuentran en Moran (1950) y la derivación de la distribución estadística del estadístico del test y su aplicación a varios modelos se describe en Kelejian, y Prucha (2001). de correlación $\geq 0,7$ ), por constituir información redundante en el modelo econométrico.

Los modelos econométricos estimados corresponden a modelos tipo logit, en los que se asume la existencia de una variable respuesta latente, $y_{i}{ }^{*}$, representada por la expresión

$$
y_{i}^{*}=\beta^{\prime} x_{i}+\mu_{i}
$$

Donde $y_{i}^{*}$ representa la renta económica, $\chi_{i}$ es un vector de variables explicadoras, $\beta$ es un vector de coeficientes a estimar y $\mu$ i es un vector de errores estocásticos. El subíndice $i$ representa la unidad de observación que en la presente investigación corresponde a un píxel de $30 \mathrm{~m}$. Dado que no es posible observar la renta económica de información de coberturas terrestres proveniente de sensores remotos (Geoghegan y otros, 2001), se usa una variable dicotómica o dummy definida como

$$
y=\left\{\begin{array}{l}
1 \text { si } y_{i}^{*}>0 \\
0 \text { en caso contrario }
\end{array}\right\} .
$$

Esto implica que un píxel se deforestará si los retornos económicos de la conversión del bosque son mayores a cero. Si se asume una función logística para la distribución acumulada de los errores $\mu i$, se obtiene un modelo logit de la forma

$$
\operatorname{Pr}(y=1, \text { deforestado })=\frac{e^{\beta^{\prime} x_{i}}}{1+e^{\beta x_{i}}} .
$$

El modelo de regresión de la ecuación (10) se estimó para Urabá con una variable dependiente en función de las potenciales variables explicadoras descritas anteriormente. Los modelos econométricos se estimaron en el software estadístico STATA ${ }^{\mathrm{TM} 9.1 .}$

Para la evaluación de los modelos econométricos estimados se usaron los siguientes criterios: 1) el valor log-likelihood; 2) el $\mathrm{R}^{2}$ ajustado de McFadden; 3) el Criterio de Información de Akaike (AIC) (Long, 1997); 4) el área bajo la curva ROC (Receiver Operating Characteristic por su sigla en inglés), que se obtiene al definir varios valores umbrales para 
considerar un píxel como deforestado y graficar la proporción de positivos ciertos (deforestación correctamente predicha) en función de falsos positivos (deforestación incorrectamente predicha). El área bajo la curva ROC representa una medida del poder de discriminación del modelo; con valores de 0,5 y 1 indica predicciones aleatorias y perfectas, respectivamente. Modelos estadísticos con valores altos de log-likelihood, $\mathrm{R}^{2}$ ajustado de McFadden y área bajo la curva ROC son deseables. Para el Criterio de Información de Akaike (AIC) son deseables los modelos estadísticos con los menores valores. Para cada píxel se estimó la probabilidad de deforestación con la siguiente ecuación

$$
P_{i}=\frac{1}{1+\exp \left(-\beta_{0}-\beta_{1} X_{1 i}-\beta_{2} X_{2 i}-\ldots-\beta_{n} X_{n i}\right)},
$$

la cual representa la función de distribución acumulada de una variable que se distribuye como una función logística. La asignación a cada píxel de una probabilidad de deforestación con el modelo econométrico seleccionado permitió obtener una superficie de probabilidades estimadas de deforestación para el área cubierta con bosques en 1980.

\section{RESULTADOS}

La deforestación observada en la zona centro y norte de la región de Urabá entre 1980 y 2000 ocurrió a ambos lados de la vía Medellín-Turbo (figura 2). En el flanco occidental de la vía se presentó en bosques de catival, manglar y bosques de mosaico catival-manglar en el municipio de Turbo. En el flanco oriental se deforestaron los bosques localizados en los municipios de Turbo y Necoclí, así como aquellos bosques intervenidos ubicados en Mutatá y otros municipios del eje bananero como Chigorodó, Carepa y Apartadó. En el área central la mayoría de los bosques se deforestaron para establecer pastos a lo largo de la vía principal, específicamente entre Mutatá y Chigorodó, y más al norte entre el Tres y San José de Mulatos-Pueblo Nuevo. Algunas áreas en bosque se transformaron a agricultura comercial cerca de Chigorodó, y entre Turbo y Apartadó.

Figura 2. Deforestación observada en la zona centro y norte de Urabá, Colombia, 1980-2000. Tamaño de píxel: $30 \mathrm{~m}$. Las líneas en el mapa representan la red

vial existente al inicio del período de análisis.

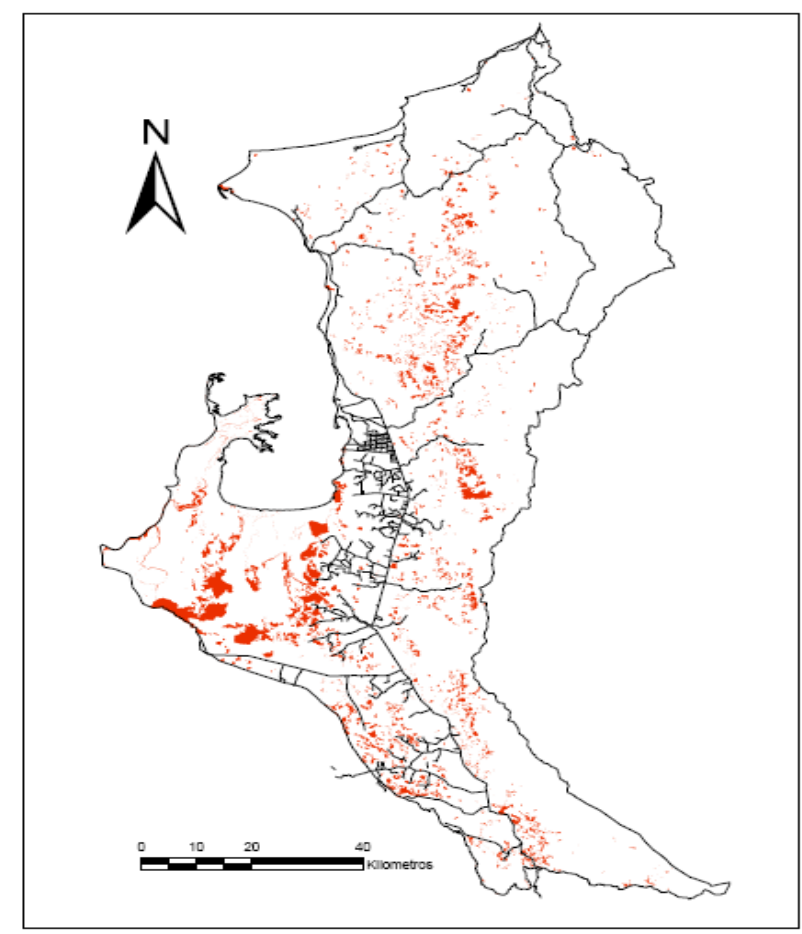

Fuente: Elaboración propia.

El cálculo del índice de Morán arrojó un valor de 0 , lo que significa que el patrón de la variable dependiente en la muestra seleccionada no está correlacionado y es aleatorio. Esto excluye la presencia de autocorrelación espacial en la variable dependiente de deforestación. La evaluación de la correlación entre pares de variables explicadoras no mostró en ningún caso un coeficiente de correlación de Pearson superior a 0,8. Cuatro modelos preliminares se estimaron en Stata ${ }^{\mathrm{TM}}$ 9.1, cuyos resultados se presentan en la tabla 1 .

Los modelos estimados ${ }^{3}$, que difieren en el tipo de variable de acceso a mercados incorporada,

\footnotetext{
3 La información utilizada en la estimación de los resultados del presente artículo está disponible para fines académicos, previa solicitud directa a los autores.
} 
fueron estadísticamente significativos según los valores de la prueba de razón de verosimilitud, cuyo estadístico se distribuye como una chi-cuadrada. No obstante, los modelos por no ser anidados no se pueden comparar directamente. Se seleccionó el modelo uno de la tabla 1, que incluye como variable explicadora la distancia ponderada a los centros de importancia regional Apartadó y Turbo, por ser estos los dos epicentros urbanos en los que se concentró la dinámica socio-económica e histórica de Urabá, durante el período de análisis de la presente investigación. Es importante mencionar que en el modelo seleccionado se incluyó la variable distancia a ríos, la cual presentó un coeficiente de correlación con la variable distancia a los centros de importancia regional de 0,73. Este valor es ligeramente superior al umbral de 0,7 definido para el análisis de multicolinealidad entre pares de variables. La inclusión de la variable distancia a ríos se explica por la influencia sustancial de los ríos navegables en la movilización de productos y personas en la región de Urabá.

Tabla 1. Resultados de los modelos econométricos logit preliminares, estimados con las variables de distancia ponderada por impedancia a diferentes centros poblados, para la región centro y norte de Urabá, 1980-2000.

\begin{tabular}{|c|c|c|c|c|}
\hline Variable & Modelo 1 & Modelo 2 & Modelo 3 & Modelo 4 \\
\hline \multirow{2}{*}{ Distancia a centros de importancia regional } & $-0,00001125^{* * *}$ & & & \\
\hline & $(2,15 \mathrm{E}-06)$ & & & \\
\hline \multirow{2}{*}{ Distancia a centros secundarios } & & $-0,0000113^{* * *}$ & & \\
\hline & & $(2,07 \mathrm{E}-06)$ & & \\
\hline \multirow{2}{*}{ Distancia a centros Básicos } & & & $-0,00000758^{* * *}$ & \\
\hline & & & $(1,96 \mathrm{E}-06)$ & \\
\hline \multirow{2}{*}{ Distancia a centros Rurales } & & & & $-0,00001446^{* * *}$ \\
\hline & & & & $(2,32 \mathrm{E}-06)$ \\
\hline \multirow{2}{*}{ Distancia a ríos (m) } & $0,000038^{*}$ & $0,000026+$ & $-0,000026^{*}$ & $-0,000014$ \\
\hline & $(1,6 \mathrm{E}-05)$ & $(1,5 \mathrm{E}-05)$ & $(1,2 \mathrm{E}-05)$ & $(1,3 \mathrm{E}-05)$ \\
\hline \multirow{2}{*}{ Áreas Naturales Protegidas } & $1,436+$ & $1,561 \mp$ & 0,428 & $2,212^{* *}$ \\
\hline & $(0,818)$ & $(0,818)$ & $(0,759)$ & $(0,854)$ \\
\hline \multirow{2}{*}{ Pendiente (grados) } & $-0,0777^{* * *}$ & $-0,0693^{* * *}$ & $-0,0692^{* * *}$ & $-0,0482 * *$ \\
\hline & $(0,016)$ & $(0,016)$ & $(0,016)$ & $(0,017)$ \\
\hline \multirow{2}{*}{ Aptitud agrícola } & $1,163^{*}$ & $1,024^{*}$ & $1,152^{*}$ & $0,959+$ \\
\hline & $(0,479)$ & $(0,497)$ & $(0,462)$ & $(0,490)$ \\
\hline \multirow{2}{*}{ Especialización plátano } & $-0,612$ & 0,282 & $-0,270$ & $-0,0004$ \\
\hline & $(0,560)$ & $(0,659)$ & $(0,569)$ & $(0,556)$ \\
\hline \multirow{2}{*}{ Especialización banano } & $-2,287$ † & $-1,686$ & $-2,010+$ & $-2,086+$ \\
\hline & $(1,181)$ & $(1,330)$ & $(1,199)$ & $(1,159)$ \\
\hline \multirow{2}{*}{ Especialización maíz } & $5,852^{*}$ & $7,624^{*}$ & $6,041^{*}$ & $6,235^{*}$ \\
\hline & $(2,814)$ & $(3,582)$ & $(2,912)$ & $(2,764)$ \\
\hline \multirow{2}{*}{ Especialización pasto } & $12,379 x$ & 14,039т & $12,928^{*}$ & $12,993^{*}$ \\
\hline & $(6,346)$ & $(7,945)$ & $(6,540)$ & $(6,231)$ \\
\hline \multirow{2}{*}{ NBI rural 1993} & $-0,403 * *$ & $-0,425^{*}$ & $-0,362^{*}$ & $-0,375^{* *}$ \\
\hline & $(0,138)$ & $(0,173)$ & $(0,142)$ & $(0,135)$ \\
\hline \multirow{2}{*}{ Tasa de crecimiento rural,1980-1990 } & 0,345 & 0,124 & 0,171 & 0,463 \\
\hline & $(0,555)$ & $(0,557)$ & $(0,559)$ & $(0,548)$ \\
\hline \multirow{2}{*}{ Tasa de crecimiento rural,1990-2000 } & $4,563^{*}$ & $4,723+$ & $4,7504^{*}$ & $4,759^{*}$ \\
\hline & $(2,057)$ & $(2,553)$ & $(2,114)$ & $(2,019)$ \\
\hline \multirow{2}{*}{ Densidad poblacional 1990 (No/km2) } & 0,0131 & 0,0136 & 0,0159 & 0,0158 \\
\hline & $(0,0108)$ & $(0,0107)$ & $(0,0114)$ & $(0,0111)$ \\
\hline \multirow{2}{*}{ Zona de vida } & $-0,936^{* *}$ & $-0,886^{* *}$ & $-1,0236^{* * *}$ & $-1,065^{* *}$ \\
\hline & $(0,314)$ & $(0,319)$ & $(0,309)$ & $(0,328)$ \\
\hline Constante & 15,901 & 11,966 & 11,306 & 10,920 \\
\hline Chi-cuadrado c2 & 389,8 & 392,7 & 371,3 & 407,1 \\
\hline AIC & 490,2 & 487,3 & 506,7 & 472,9 \\
\hline $\mathrm{ROC}$ & 0,911 & 0,912 & 0,902 & 0,922 \\
\hline R2-McFadden & 0,423 & 0,427 & 0,402 & 0,444 \\
\hline Log-Likelihood & $-230,1$ & $-228,7$ & $-239,4$ & $-221,5$ \\
\hline
\end{tabular}

Nota: Valores de significancia $\pm \mathrm{p}<.1 ;^{*} \mathrm{p}<.05 ;{ }^{* *} \mathrm{p}<.01 ;{ }^{* * *} \mathrm{p}<.001$. Los valores en paréntesis corresponden a errores estándar. Fuente: Elaboración propia 
El modelo seleccionado contiene varias variables explicadoras, por lo que las correlaciones simples entre pares de ellas no detectan la posible correlación entre más de dos variables (Maddala, 1996). Por tanto, se usó el número de condición, el cual mide la robustez de los estimadores de regresión a pequeños cambios en los datos (Maddala, 1996). Para el cálculo del número de condición las variables se escalaron a una unidad de longitud, pero no se centraron, dada la posibilidad de ocultar la colinealidad con el término constante de la regresión (Kennedy, 2003). En la literatura especializada se suele afirmar que un número de condición mayor a 30 indica la existencia de fuerte colinealidad (Kennedy, 2003). El número de condición de las variables explicadoras fue 55,3; por ello, se procedió a examinar cuidadosamente tanto la significancia estadística individual como el signo de cada una de las variables en el modelo econométrico seleccionado.

Mediante un procedimiento iterativo, se excluyeron variables con una baja significancia estadística individual o con correlaciones simples significativas. Las variables excluidas fueron la tasa de crecimiento de la población rural 1980-1990, por baja significancia estadística y correlación con la tasa de crecimiento de la población rural 1990-2000. También se excluyó la variable especialización en plátano por constituir información contenida en la variable especialización en banano, el cual es también un producto de exportación. Finalmente, aquellas variables explicadoras cuyos coeficientes tuvieron signos que no correspondieron con el teóricamente esperado se excluyeron. Esto podría explicarse por el cálculo de las variables con información relativamente gruesa como fue el caso de la zona de vida (escala 1:500.000), o a una escala municipal con poca discriminación local como el índice NBI rural 1993, o por tratarse de una superficie pequeña de áreas protegidas $\left(\sim 832 \mathrm{~km}^{2}\right)$ que corresponde $\sim 10 \%$ de la superficie total del área de estudio. En la tabla 2 se presentan los resultados del modelo econométrico final, cuyo número de condición7,9 no sugiere multicolinealidad entre las variables regresoras.

Tabla 2. Resultados del modelo econométrico logit seleccionado de deforestación para la zona centro y norte de la región de Urabá, Colombia, 1980-2000.

\begin{tabular}{lcc}
\hline \multicolumn{1}{c}{ Variable } & Parámetro & Elasticidad \\
\hline Distancia a centros & $-8,92 \mathrm{E}-06^{* * *}$ & -1.062 \\
de importancia regional & $(1,67 \mathrm{E}-06)$ & $(0,240)$ \\
& $3,64 \mathrm{E}-05 * *$ & 0,360 \\
Distancia a ríos (m) & $(1,28 \mathrm{E}-05)$ & $(0,137)$ \\
& $-0,0711^{* * *}$ & $-0,354$ \\
Pendiente (grados) & $(0,015)$ & $(0,088)$ \\
& $1,044^{*}$ & 0,046 \\
Aptitud agrícola & $(0,474)$ & $(0,021)$ \\
& $0,527 \mp$ & 0,201 \\
Especialización en & $(0,315)$ & $(0,115)$ \\
banano & $2,756^{* * *}$ & 1,214 \\
& $(0,623)$ & $(0,258)$ \\
Especialización en maíz & $3,078^{* *}$ & 1,662 \\
& $(1,053)$ & $(0,484)$ \\
Especialización en pasto & $0,690 * *$ & $-0,050$ \\
Tasa de crecimiento & $(0,255)$ & $(0,016)$ \\
rural, 1990-2000 & $0,023 *$ & 0,263 \\
Densidad poblacional & $(0,009)$ & $(0,115)$ \\
1990 (No/km2) & $-5,047 * *$ & \\
Constante & $(1,783)$ & \\
\hline
\end{tabular}

Chi-cuadrado X2

370,47

AIC 499,601

ROC $\quad 0,898$

R2-McFadden $\quad 0,41$

Log-Likelihood $\quad-239,801$

Notas: Valores de significancia: ${ }^{+} \mathrm{p}<0,10$; $^{*} \mathrm{p}<0,05 ;{ }^{* *} \mathrm{p}<0,01$; ${ }^{* * *}$ $p<0,001$. Los valores en paréntesis corresponden a errores estándar. La mayoría de los valores de las elasticidades son estadísticamente significativos a un nivel por lo menos de 0,05. La excepción es la variable especialización productiva en banano, cuya elasticidad es estadísticamente significativa a un nivel de 0,10.

Fuente: elaboración propia

Los signos de las variables distancia ponderada por la impedancia, pendiente, aptitud agrícola, especialización en banano, especialización en maíz, especialización en pasto y la tasa de crecimiento rural coinciden con los signos esperados de manera 
anticipada. Una mayor distancia a centros de importancia regional, así como una mayor pendiente implican una menor deforestación. Por el contrario, la deforestación aumenta en áreas con mayor especialización productiva, mejor aptitud agrícola, más densidad poblacional y mayor tasa de crecimiento de la población rural 1990-2000. El coeficiente de la variable distancia a ríos no posee el signo esperado. Sin embargo, ello podría explicarse por la configuración espacial tan característica de los ríos en el área de estudio. Los dos ríos navegables en la región, Atrato y León, corren relativamente paralelos en dirección sur-norte hacia el Golfo de Urabá, por lo que alejarse en sentido oriente-occidente de un río implica acercarse al otro.

Los mayores errores estándar los presentan las variables de especialización y la tasa de crecimiento poblacional rural. Ello podría explicarse por el cálculo de estas variables a escala municipal. La magnitud de los parámetros estimados y los valores de elasticidades sugieren que los principales determinantes de la deforestación en las zonas centro y norte de Urabá fueron la especialización en pasto, la especialización en maíz, la distancia ponderada por la impedancia a los centros de importancia regional y la distancia a los principales ríos de la región. Los parámetros con menor valor en magnitud corresponden a la densidad poblacional en 1990, una proxy del número de familias agrícolas, así como la distancia ponderada por la impedancia a los centros de importancia regional y a los principales ríos de la región, que se asumen como proxies de accesibilidad a mercados. El área bajo la curva ROC fue 0,89, lo que sugiere un excelente poder de discriminación del modelo econométrico.

El modelo final se empleó para obtener una superficie continua de probabilidades estimadas de deforestación para el área cubierta por bosques en 1980 (figura 3). El modelo predice bajas probabilidades de deforestación para la zona sureste de la Serranía de Abibe, estribaciones del Parque Nacional Natural de Paramillo. Esta área es remota, con altas pendientes, baja densidad vial, donde se observó la menor conversión de bosques en el período 1980-2000. Altas probabilidades de deforestación se predicen para la zona norte, entre los corregimientos El Dos y Trinidad, así como para el Golfo de Urabá, en bosques de catival y manglar próximos al área de consolidación de la producción comercial de banano. La existencia de dos vías fluviales navegables podría explicar la significativa reducción de los bosques en el Golfo de Urabá.

Figura 3. Superficie de probabilidades estimadas de deforestación en la zona centro y norte de Urabá, 1980-2000. Tamaño de píxel: 30 m.

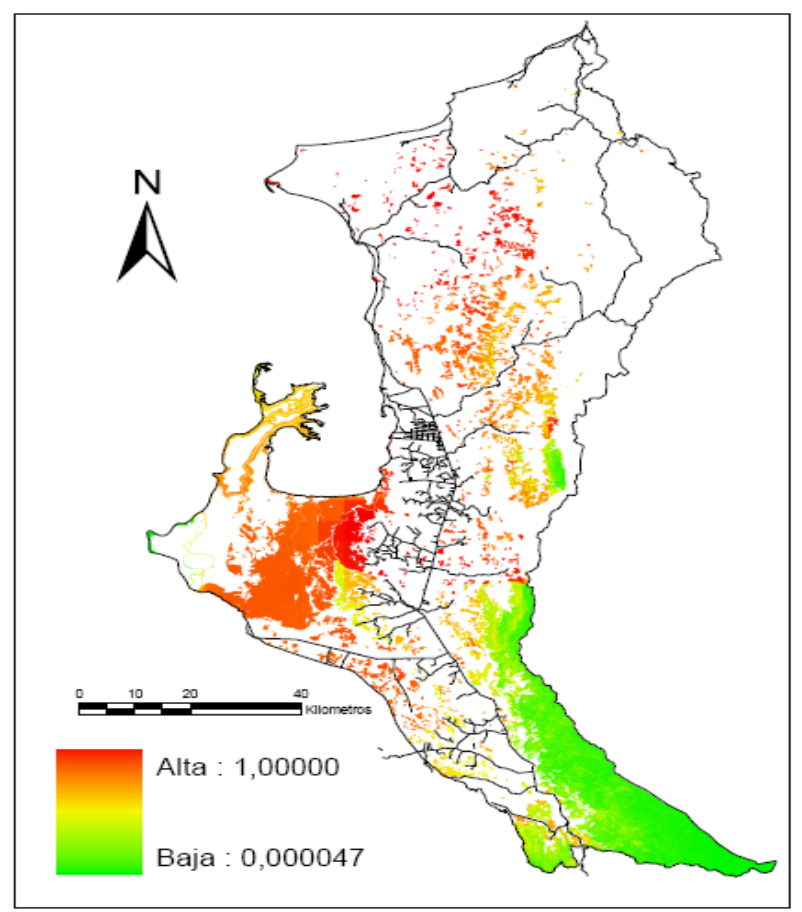

Fuente: Elaboración propia.

\section{DISCUSIÓN}

El modelo econométrico para el estudio de la deforestación observada en Urabá, en el período 1980 -2000, contiene nueve variables: distancia a centros de importancia regional, cuyo cálculo es consistente con lo realizado en otros estudios (Chomitz y Gray, 1996; Nelson y Hellerstein, 1997; Blackman y otros, 2003); distancia a ríos; pendiente; 
aptitud agrícola; coeficientes de especialización en banano, maíz y pasto; tasa de crecimiento de la población rural 1990-2000, y densidad poblacional 1990. Las variables son similares a aquellas incluidas en estudios econométricos previos de deforestación tropical, con excepción de las variables de especialización productiva.

Un valor de 0,897 para el área bajo la curva ROC (tabla 2), representa una excelente medida de la capacidad de discriminación del modelo estimado (Jurkowski y otros, 2005). El valor está lejos de 0,5 , que equivale a una habilidad aleatoria de discriminación del modelo. En lo que respecta al $R^{2}$ sugerido por McFadden, un valor de 0,41 se considera muy satisfactorio en modelos discretos y datos de corte transversal. Las variables incluidas en el modelo explican $41 \%$ de la variación observada en la probabilidad de deforestación, lo cual difiere sustancialmente de una variación explicada del 22\% para el modelo de deforestación en el departamento de Antioquia (Orrego, 2009). Si se tiene en cuenta que la aproximación de modelación, el tamaño de la unidad de análisis (píxel de $30 \mathrm{~m}$ ) y el modelo estadístico son similares, el mejor ajuste estadístico del modelo para Urabá se podría explicar por la inclusión de variables como especialización productiva y dinámica poblacional. Estas no se incluyeron en el modelo de deforestación para Antioquia por ausencia de información.

De acuerdo con la magnitud de los parámetros y el valor de elasticidades, la especialización en pasto, la especialización en maíz, la distancia ponderada por la impedancia a los centros de importancia regional y la distancia a los principales ríos de la región fueron los principales determinantes de la deforestación en Urabá. Los valores de elasticidades tienden a ser menores a uno, similar a lo obtenido en el estudio de la deforestación en Antioquia (Orrego, 2009), en el que solo la variable índice de Gini, una medida de la concentración de la propiedad de la tierra rural, presentó una elasticidad mayor a uno. En Urabá la especialización en maíz, la especialización en pasto y la distancia ponderada por la impedancia a los centros de importancia regional presentaron elasticidades mayores a uno en valor absoluto.

La variable distancia a ríos fue estadísticamente significativa a un nivel de 0,01. Ello difiere del modelo de deforestación para Antioquia (Orrego, 2009), así como de resultados de otros modelos regionales de deforestación estimados para Colombia (Etter y otros, 2006d). El modelo de deforestación de Urabá podría capturar la influencia que ejercen los ríos en la dinámica socio-económica de la región. Allí los ríos Atrato y León facilitan la movilización de personas y productos, entre los que se incluyen maderas con valor comercial. Los índices de especialización productiva en pasto, banano y maíz parecen capturar la compleja imbricación y coexistencia del uso ganadero en la mayor parte del territorio, la consolidación de un enclave de producción de banano, y la influencia de economías campesinas dedicadas a la producción de cultivos de subsistencia.

Aunque las variables especialización en pasto y en maíz se calcularon para cada municipio, estas variables poseen el signo positivo teóricamente esperado. Ello refleja el efecto de la ganadería y la economía campesina sobre la deforestación en Urabá. El valor de elasticidad de la variable especialización en pasto (tabla 2), constituye una evidencia empírica de la expansión de la actividad ganadera a expensas de la conversión de bosques. La significancia estadística de la variable especialización en maíz probablemente captura la expansión de la frontera agrícola. Inicialmente un proceso de colonización espontánea conduce a la desaparición de bosques con la arraigada práctica de tala y quema por colonos. Ellos extraen árboles maderables con valor comercial y luego queman el material vegetal aéreo remanente. Luego se establecen cultivos de maíz, yuca, arroz y plátano para satisfacer necesidades familiares, con excepción del maíz cuyos excedentes de producción se venden en mercados locales. Posteriormente, la economía campesina se articula con la producción pecuaria 
mediante la siembra de pastos y el establecimiento de relaciones comerciales con otras personas para la cría y el levante de ganado. Este proceso colonocampesino-colono se repite continuamente.

La aptitud agrícola sugiere un efecto positivo sobre la deforestación (tabla 2). Es decir, aquellas áreas con mayor aptitud natural para la agricultura tienden a exhibir una mayor deforestación, lo que concuerda con los resultados del estudio de deforestación en Santa Cruz, Bolivia (Mertens y otros, 2004). Las clases agrológicas con mayor aptitud agrícola fueron las III y IV, con suelos planos y fértiles. En estas áreas tiende a consolidarse la producción comercial de banano. Este resultado es consistente con estudios en los que se señala a la expansión de la agricultura como una causa significativa de deforestación tropical (Geist y Lambin, 2002; Gibbs y otros, 2010). La pendiente fue estadísticamente significativa $(p<0,001)$ y se correlacionó negativamente con la deforestación (tabla 2), como ocurrió en estudios previos (Chomitz y Gray, 1996; Cropper, Puri y Griffiths, 2001) en los que se sugiere que bosques ubicados en sitios con altas pendientes serían menos susceptibles a deforestarse. Ello se podría explicar por los altos costos de conversión de tierras con topografía abrupta o escarpada.

El desplazamiento forzado no se incluyó explícitamente como variable en el modelo, por diferencia en los datos luego de compilar la información reportada en las fuentes de información consultadas. No obstante, se asume que la tasa de crecimiento de la población rural podría ser una variable proxy para el desplazamiento forzado, originado bien sea por disputas por la tierra o por el conflicto armado. La tasa de crecimiento de la población rural 1990-2000 fue estadísticamente más significativa que la tasa para el período 1980-1990 (tabla 1). Ello sugiere que el efecto de la población sobre la deforestación fue más significativo en la segunda década del período de análisis.

En Urabá, la población constituye un factor muy dinámico. Los flujos migratorios se acentuaron en las décadas de 1950 y 1960, con el establecimiento de empresas extranjeras dedicadas a la producción de banano. Así la región se convirtió en destino geográfico de colonos provenientes del interior de Antioquia seducidos por la existencia de empleo en cultivos de banano. En la década de 1970 se produjo otro movimiento migratorio que desalojó a colonos localizados a lo largo del denominado eje bananero, y los forzó a desplazarse a áreas remotas como las estribaciones de la Serranía de Abibe (Departamento Nacional de Planeación y Gobernación de Antioquia, 2006).

Aunque la población creciente de colonos y pequeños agricultores con limitados recursos se señala como un factor de presión antrópica sobre los bosques en Colombia (Etter y otros, 2006d), el crecimiento de la población rural no fue el principal determinante de la deforestación en Urabá en el período de estudio. Este resultado coincide con un estudio de deforestación en Brasil (Pfaff, 1999), en el que la importancia relativa de variables demográficas se atenuó luego de incorporar en el modelo otras variables explicadoras de la deforestación.

Dos aspectos estadísticos del modelo econométrico merecen especial atención. Ellos son el control de la correlación espacial de la variable de deforestación y la potencial endogeneidad entre las vías y agricultura. La autocorrelación espacial se controló satisfactoriamente con la estrategia de muestreo empleada. El índice de Morán arrojó un valor de 0 , lo que indica que el patrón espacial de la variable dependiente es aleatorio. La endogeneidad, por su parte, se controló al incluir en el modelo una variable como la aptitud agrícola, la cual explica la heterogeneidad de la calidad del suelo. Además, la endogeneidad entre vías y agricultura no existe si se asume que el trazado de las vías no es influenciado explícitamente por la necesidad de cruzar sitios con potencial de desarrollo agrícola. Este parece ser el caso en Urabá donde, de manera similar a otros estudios (Mertens y Lambin, 2000), la construcción 
de las vías tuvo el propósito de conectar sitios remotos a mercados nacionales.

El satisfactorio ajuste estadístico del modelo de deforestación para Urabá podría deberse a la calidad de la información de coberturas terrestres que se empleó. Esta proviene fundamentalmente de fotografías aéreas, las cuales permiten una discriminación de coberturas terrestres con una menor confusión que la clasificación obtenida de imágenes de satélite en áreas tropicales. No obstante, existen algunas limitaciones del estudio. El modelo combina variables calculadas con información original que posee distintas resoluciones, desde píxel a municipio. El modelo estimado no incorpora explícitamente, por falta de información, variables que constituyen importantes determinantes económicos de la deforestación, como las motivaciones de los propietarios de la tierra, los precios de la tierra, de productos y de insumos. Otra posible limitación del modelo consiste en la no incorporación explícita de variables que den cuenta de las rentas de usos alternativos de la tierra. Ello no permite simular el efecto sobre el uso de la tierra de políticas públicas basadas en incentivos como subsidios a la producción agrícola o impuestos a la deforestación.

\section{CONCLUSIONES}

Un modelo económico teórico de conversión de uso de la tierra rural junto con un modelo econométrico fueron empleados para identificar los principales determinantes y su importancia relativa en la deforestación observada en las zonas centro y norte de Urabá, Colombia, en el período 1980-2000. El modelo econométrico seleccionado describe satisfactoriamente la compleja interacción de causas próximas y subyacentes que explican la deforestación en la región de Urabá, y contiene nueve variables explicadoras: la distancia ponderada a los centros de importancia regional (Apartadó y Turbo); la distancia a ríos; la pendiente; la aptitud agrícola; la tasa de crecimiento rural 1990-2000; la densidad poblacional en 1990; así como tres índices de especialización productiva en banano, maíz y pasto. Las variables se consideran como proxies que afectan bien sea la demanda o la oferta por el factor de producción tierra sin bosque.

La magnitud de los parámetros y el valor de elasticidades permiten concluir que la especialización en pasto, la especialización en maíz, la distancia ponderada por la impedancia a los centros de importancia regional y la distancia a los principales ríos de la región fueron los principales determinantes de la deforestación en las zonas centro y norte de Urabá. Estos determinantes corresponden a las interacciones entre causas tanto próximas como subyacentes, relacionados con la extensión de la infraestructura, la expansión de la agricultura comercial, la fertilidad del suelo y dinámicas demográficas. De esta manera, en la región de Urabá parece ser que coexisten múltiples causas de deforestación que han estado íntimamente relacionadas. Ello contrasta con patrones temporales globales de deforestación (Rudel y otros, 2009; Defries y otros, 2010), en los que determinantes de conversión de bosques tropicales, asociados con colonizaciones dirigidas y crecimiento de la población rural, se sustituyeron por la exportación de productos agrícolas y el crecimiento de la población urbana.

\section{BIBLIOGRAFÍA}

Aguiar A., p. D.; Câmara, G. y Escada S., M. I. (2007). Spatial Statistical Analysis of Land-Use Determinants in the Brazilian Amazonia: Exploring IntraRegional Heterogeneity. En: Ecological Modelling, Vol. 209, N²-4, p. 169-188.

Armenteras, D.; Rudas, G.; Rodriguez, N.; Sua, S. y Romero, M. (2006). Patterns and Causes of Deforestation in the Colombian Amazon. En: Ecological Indicators, Vol. 6, p. 353-368.

Bell, K. p. y Bockstael, N. E. (2000). Applying the Generalized-Moments Estimation Approach to Spatial Problems Involving Microlevel Data. En: The Review of Economics and Statistics, Vol. 82, $\mathrm{N}^{\circ}$ 1, p. 72-82. 
Blackman, A.; Albers, H.; Avalos-Sartorio, B. y Crooks, L. (2003). Land Cover in a Managed Forest Ecosystem: Mexican Shade Coffee. Resources for the Future, 34p.

Blackman, A.; Albers, H.; Avalos-Sartorio, B. y Murphy, L. C. (2007). Land Cover in a Managed Forest Ecosystem: Mexican Shade Coffee. Resources for the Future, 36p.

Carrión-Flores, C. y Irwin, E. G. (2004). Determinants of Residential Land-Use Conversion and Sprawl at the Rural-Urban Fringe. En: American Journal of Agricultural Economics, Vol. 86, N 4, p. 889-904.

CIESIN-CIAT. (2005). Gridded Population of the World Version 3 (GPW V3). [En línea] Socioeconomic Data and Applications Center (SEDAC), Columbia University, Palisades, New York, Disponible en: http://sedac.ciesin.columbia.edu/gpw. [Enero 25 de 2010].

Corpourabá. (1984). Plan de Desarrollo de Urabá. Diversificación y Bienestar hacia la industrialización. Medellín: Departamento Nacional de Planeación. Corporación Regional de Desarrollo de Urabá, 368p.

Cropper, M.; Griffiths, C.y Mani, M. (1999). Roads, Population, Pressures, and Deforestation in Thailand, 1976-1989. En: Land Economics, Vol. $75, \mathrm{~N}^{\circ} 1$, p. $58-73$.

Cropper, M.; Puri, J. y Griffiths, C. (2001). Predicting the Location of Deforestation: The Role of Roads and Protected Areas in North Thailand. En: Land Economics, Vol. 77, N 2, p. 172-186.

Chomitz, K. M. y Gray, D. A. (1996). Roads, Land Use, and Deforestation: A Spatial Model Applied to Belize. En: The World Bank Economic Review, Vol. 10, $N^{\circ} 3$, p. 487-512.

Chomitz, K. M. y Thomas, T. S. (2003). Determinants of Land Use in Amazonia: A Fine-Scale Spatial Analysis. En: American Journal of Agricultural Economics, Vol. 85, N 4, p. 1016-1028.

Defries, R.; Rudel, T.; Uriarte, M. y Hansen, M. (2010). Deforestation Driven by Urban Population Growth and Agricultural Trade in the Twenty-First Century. En: Nature Geoscience, Vol. 3, marzo, p. 178-181.
Departamento Administrativo de Planeación y Gobernación de Antioquia (2007). Anuario Estadístico de Antioquia. Medellín.

Departamento Nacional de Planeación y Gobernación de Antioquia (2006). Plan Estratégico para la Región de Urabá-Darién. Primera fase: Construcción de insumos para el proceso en la región. Departamento Nacional de Planeación - Dirección de Desarrollo Territorial Sostenible - Subdirección de Ordenamiento y Desarrollo Territorial. Departamento Administrativo de Planeación de Antioquia. Dirección de Planeación Estratégica Integral. Junta Efemérides Urabá, $154 p$.

Etter, A.; Mcalpine, C.; Phinn, S.; Pullar, D. y Possingham, H. (2006a). Characterizing a Tropical Deforestation Wave: A Dynamic Spatial Analysis of a Deforestation Hotspot in the Colombian Amazon. En: Global Change Biology, Vol. 12, p. 1409-1420.

Etter, A.; Mcalpine, C.; Phinn, S.; Pullar, D. yPossingham, H. (2006b). Unplanned Land Clearing of Colombia Rainforests: Spread like Disease? En: Landscape and Urban Planning, Vol. 77, p. 240-254.

Etter, A.; Mcalpine, C.; Pullar, D. y Possingham, H. (2006c). Modelling the Conversion of Colombia Lowland Ecosystems since 1940: Drivers, Patterns and Rates. En: Journal of Environmental Management, Vol. 79, p. 74-87.

Etter, A.; Mcalpine, C.; Wilson, K.; Phinn, S. y Possingham, H. (2006d). Regional Patterns of Agricultural Land Use and Deforestation in Colombia. En: Agriculture, Ecosystems and Environment, Vol. 114 , p. 369-386.

Geist, H. J. y Lambin, E. F. (2002). Proximate Causes and Underlying Driving Forces of Tropical Deforestation. En: BioScience, Vol. 52, N 2, p. 143-150.

Geoghegan, J.; Villar, S. C.; Klepeis, P.; Mendoza, p. M.; Ogneva-Himmelberger, Y.; Chowdhury, R. R.;Turner Ii, B. L. y Vance, C. (2001). Modeling Tropical Deforestation in the Southern Yucatán Peninsular Region: Comparing Survey and Satellite Data. En: Agriculture, Ecosystems and Environment, Vol. 85, p. $25-46$. 
Gibbs, H. K.; Ruesch, A. S.; Achard, F.; Clayton, M. K.; Holmgren, P.; Ramankutty, N. y Foley, J. A. (2010). Tropical Forests Were the Primary Sources of New Agricultural Land in the 1980s and 1990s. En: Proceedings of the National Academy of Sciences (Early Edition, September 2010).

Gobernación de Antioquia. (2000). Anuario Estadístico de Antioquia, Medellín.

Hijmans, R. J.; Cameron, S. E.; Parra, J. L.; Jones, p. G. y Jarvis, A. (2005). Very High Resolution Interpolated Climate Surfaces for Global Land Areas. En: International Journal of Climatology, Vol. 25, p. 1965-1978.

Jurkowski, P.; Ćwiklinska-Jurkowska, M.; Doniec, Z. y Szaflarska-Poplawska, A. (2005). Receiver Operating Characteristic (ROC) and other Curves Measuring Discriminability of Classifiers' Ensemble for Asthma Diagnosis. En: Advances in Medical Sciences, Vol. 50, N 2, p. 65-67.

Kaimowitz, D. y Angelsen, A. (1998). Economic Models of Tropical Deforestation: A Review. Center for International Forestry Research, Bogor, Indonesia.

Kelejian, H. y Prucha, I. (2001). On the Asymptotic Distribution of the Moran I Test Statistic with Applications. En: Journal of Econometrics, Vol. $104, N^{\circ} 2$, p. 219-257.

Kennedy, p. (2003). A Guide to Econometrics. Cambridge, Massachusetts, MIT Press, 623p.

Klingebiel, A. y Montgomery, p. (1961). Land-Capability Classification. En: Agriculture Handbook. Vol. 210, 21p. [En línea] Soil Conservation Service, U.S. Dept. of Agriculture.Natural Resources Conservation Service, Disponible en: http://naldr.nal.usda.gov/NALWeb/Agricola__ Link. asp?Accession=CAT10. [Enero 25 de 2010].

Long, J. S. (1997). Regression Models for Categorical and Limited Dependent Variables. Thousand Oaks, California: SAGE Publications, Inc., 296p.

Lubowski, R. N.; Bucholtz, S.;Claassen, R.;Roberts, M. J.;Cooper, J. C.; Gueorguieva, A. y Johansson, R. (2006). Environmental Effects of Agricultural LandUse Change: The Role of Economics and Policy.
U.S. Dept. of Agriculture, Economic Research Service.

Maddala, G. S. (1996). Introducción a la Econometría. México, D.F: Prentice Hall, 713p.

Mertens, B. y Lambin, E. F. (2000). Land-Cover-Change Trajectories in Southern Cameroon. En: Annals of the Association of American Geographers, Vol. 90, $\mathrm{N}^{\circ}$ 3, p. 467-494.

Mertens, B.; Kaimowitz, D.; Puntodewo, A.; Vanclay, J. y Mendez, p. (2004). Modeling Deforestation at Distinct Geographic Scales and Time Periods in Santa Cruz, Bolivia. En: International Regional Science Review, Vol. 27, N 3, p. 271-296.

Moran, p. A. (1950). A Test for the Serial Independence of Residuals. En: Biometrika. Vol. 37, $\mathrm{N}^{\circ} 1 / 2$, p. $178-181$.

Munroe, D. K.; Southworth, J. y Tucker, C. M. (2002). The Dynamics of Land-Cover Change in Western Honduras: Exploring Spatial and Temporal Complexity. En: Agricultural Economics, Vol. 27, p. 355-369.

Nelson, G. y Hellerstein, D. (1997). Do Roads Cause Deforestation? Using Satellite Images in Econometric Analysis of Land Use. En: American Journal of Agricultural Economics, Vol. 79, p. 80-88.

Nelson, G.; Harris, V.;Stone, S. y Pinto, A. D. (2001). Land Use and Road Improvements: A Spatial Econometric Analysis. University of Illinois, Urbana-Champaign.

Nelson, G.; Harris, V.; Stone, S. y Pinto, A. D. (2004). Land Use and Road Improvements: A Spatial Perspective. En: International Regional Science Review, Vol. 27, N 3, p. 297-325.

Orrego, S. A. (2009). Economic Modeling of Tropical Deforestation in Antioquia (Colombia), 1980-2000: An Analysis at a Semi-Fine Scale with Spatially Explicit Data. PhD Dissertation, Oregon State University, 137p.

Pfaff A., S. p. (1999). What Drives Deforestation in the Brazilian Amazon? En: Journal of Environmental Economics and Management, Vol. 37, p. 26-43. 
Rudel, T.; Defries, R.; Asner, G. y Laurance, W. F. (2009). Changing Drivers of Deforestation and New Opportunities for Conservation. En: Conservation Biology, Vol. 23, № 6, p. 1396-1405.

Usma, J. A. (1992). Diagnóstico y Recomendaciones para el Sector Agropecuario de Urabá: Un Enfoque Espacial. Universidad de Antioquia. Facultad de Ciencias Humanas y Económicas, Medellín.
Van der Werf, G.; Morton, D.; DeFries, R.; Olivier, J.; Kasibhatla, P.; Jackson, R.; Collatz, G. y Randerson, J. (2009). $\mathrm{CO}_{2}$ Emissions from Forest Loss. En: Nature Geoscience, Vol. 2, p. 737-738.

Werner, M. (2001). Shuttle Radar Topography Mission (SRTM), Mission Overview. En: J. Telecom. (Frequenz), Vol. 55, p. 75-79. 
